\title{
A New Directional Relation Model
}

\author{
Ke Zhang, Tao Liu, Zhong Li and Wei Zhao \\ Department of Electronic and Communication Engineering \\ North China Electric Power University, Baoding, China \\ Zhangke41616@126.com
}

\begin{abstract}
Formal model of spatial directional relation is one of the most important parts in the area of spatial relation, and it is necessary to develop a new model with high generality and practicality. Firstly this paper analyzes the characteristics and disadvantages of the present models. Secondly it introduces in detail the basic notion and construction method of quadtree histogram。Thirdly a new approach to judging basic and special spatial directional relations based on quadtree histogram is proposed. Finally in order to prove the correctness of this model, several examples are given. The experimental results show that this model is feasible.
\end{abstract}

Keywords: Spatial Directional Relation; Spatial Directional Relation; Quad-tree Histogram

\section{Introduction}

As the description for human space perception is a basic ability of human cognition, it is an important basic task for computer audio-visual information processing to build automatically the description of spatial relation between objects in the image. Spatial relation is an important interdisciplinary research content related to computer science, cognitive science, cartography and geography, and it has a broad application prospect in many systems such as GIS, computer vision, human-robot interaction, medical imaging and so on.

Spatial relation is mainly constituted of directional relation, topological relation and distance relation. The fundamental theories of topology relation and distance relation are comparatively perfect, but the fundamental theories of directional relation have not any unified standard. There are many formal models of directional relation [1-19] now, but these models have many problems. So the establishment of common and practical model of directional relation is essential.

Section 2 proposes the notion of directional relation and analyzes some existing models. The model of directional relation based on Quadtree Histogram is proposed in Section 3, and tested on synthetic images in Section 4. Concluding remarks are given in Section 5.

\section{Spatial Directional Relation}

\subsection{The Notion of Directional Relation}

Directional relation is referred to the position observed by an observer in a reference framework with an observation angle between one object (target object $A$ ) and another object (reference object $B$ ). Directional relation reflects the ordering relation between spatial objects. 
It is expressed by the expression $\operatorname{Dir}(A, B, F, \theta)$. In this expression "Dir" expresses the directional relation of target object $A$ relative to reference object $B, F$ is a reference framework, and $\theta$ is an observation angle.

In this paper, directional relation is divided into the basic directional relation and the special directional relation. The basic directional relation is the directional relation that can be described by basic directional terms (above, below, left, right and so on) or combined directional terms (above-left, loosely above-right and so on) The special directional relation is the directional relation that can't be described by one or two basic directional terms, such as surround, between, etc.

\subsection{Existing Formal Models of Directional Relation}

Directional relation plays an important role in interpretation of a scene, and several formal models of assessing directional relation have been proposed. There are five main approaches for assessing the basic directional relation. The first one is based on cone model $[1,2]$ which assimilates an object to the centroid, but it doesn't take into account the effects of shape, size and distance information to directional relation. The second one is based on projection model such as 2D strings [3], minimum bounding rectangles [4], directional relation matrix [5] and so on, but these models almost assimilate an object to the rectangle, so the shape, size and distance information also can't be taken into account. The third one is based on Voronoi diagram [6] which thinks the factors affecting basic directional relation are convex shell diameter and visual area of objects, so the visual area can be regarded as research object. This model proposed that there are more than one directional line between two objects, so we should use several directional lines for describing basic directional relation. But this model has high computational complexity and gets wrong results in some cases. The forth one $[7,8]$ is based on fuzzy model which thinks directional relation has uncertainty, and this model divides the directional notions by fuzzy sets for meeting the habit of human. The fifth one is based on angle-histogram model $[9,10]$. Given a target object $A$ and a reference object $B$, the angle-histogram is computed from the angles between any two points in both objects and normalized by the maximum frequency. This histogram represents the directional relation of the target object $A$ with respect to the reference object $B$. This model takes into account shape, size and distance information, but it has high computational complexity, so it is not practical. Numerous studies are based on this notion of angle-histogram, e.g., [11-16]. In [11-13], Rhistogram, $\mathrm{R}^{*}$-histogram and F-histogram are proposed, and they can reduce the computational complexity. In [14], V-histogram is proposed in observer reference framework. In $[15,16]$, allen- histogram and DRS-histogram are proposed for handling both topological relation and directional relation. However, all these histogram models still have high computational complexity, and the judgment approaches of basic directional relation based on histogram have some disadvantages.

There are two main approaches for assessing the special directional relation. The first approach $[10,17]$ uses the fact that according to most families of directional relations, an object can be in many directions with respect to another. For example, knowing that the object $A$ is somewhat above, below, to the right and to the left of the object $B$ as well, one could conclude that the object $A$ surrounds the object $B$. But the directional relation has semantic inverse principle, so there is no way to know which one surrounds (or includes) the other. The second approach derives from Rosenfeld's visual surroundedness [18]. In [9, 10], it supposes the object $A$ is connected and does not intersect the object $B$. For any pixel $P$ of the object $B$, let $\theta_{P}$ be the angle made by the two tangents from $P$ to $A$. To each element $\theta$ of $[0,2 \pi]$, the histogram associates the number of pixels $P$ such that $\theta_{P}$ is equal to $\theta$. Finally, membership values are calculated by fuzzy sets approach. However, the computational 
complexity is very high because of calculating angle-histogram, and this method can't handle disconnected objects. In [19, 20], F-histogram is used for accessing special directional relation, but it is only suitable to the object with regular shape or small size.

In this paper, a new model of spatial directional relation is proposed. This model is based on quadtree histogram, and constructs the judgment approaches of basic and special directional relation. The results of the experiment show that it can overcome the shortcomings of the other models.

\section{The Model of Spatial Directional Relation based on Quadtree Histogram}

\subsection{Quad-tree Histogram}

Because angle-histogram considers the set of all point pairs, the computational complexity is very high. Take two-dimensional raster image for example, the computational complexity in $[9,10]$ is $\mathrm{O}\left(N^{2}\right), N$ is the number of image pixels. The computational complexity in [21] by FFT can drop to $\mathrm{O}(N \log N)$. But when the objects have too many pixels, the computation time is still very long. So the practicability of these models is reduced, we should propose a new simple histogram model.

The article [6] indicates that people firstly integrate graphics when they judge the directional relation. The level of integration is proportional to the diameter $D$ of convex hull of two objects. The article [11] indicates that cone model is suitable to the directional relation judgment of simple and regular objects. The longer the distance of two objects is, the better the effect of judgment is. In this paper the above two ideas are come down to two factors affecting the integration of graphics. 1) The longer the distance between one object and another object, the greater the possibility of integration to a point is. 2) The simpler the shape of the object is, the greater the possibility of integration to a point is.

Based on the above two factors, this paper presents quadtree histogram. The main idea of this histogram includes three steps. Firstly the objects are divided into a number of simple and regular sub-objects by quadtree algorithm, so that these sub-objects can get the standard of integration to a point. Secondly, we use the centroids of these sub-objects instead of the subobjects themselves. Thirdly, we calculate the weighted angle histogram of centroid point pairs.

In this paper we use the square as the basic graphic of sub-objects, because the square is the representational simple graphics, and facilitates the implementation of the quadtree algorithm. Firstly, we get the minimum bounding squares $\mathrm{MBS}^{B}$ and $\mathrm{MBS}^{A}$ of the reference object $B$ and the target object $A$. Secondly, we divide $\mathrm{MBS}^{B}$ and $\mathrm{MBS}^{A}$ into several subsquares $\mathrm{MBS}_{j}^{B}$ and $\mathrm{MBS}_{i}^{A}$ by quadtree algorithm and these sub-squares must meet certain conditions. Based on above two factors we define the following conditions.

1) The ratio of the side length $m$ of sub-square to the side length $M$ of the minimum bounding square $\mathrm{MBS}^{A B}$ should be less than the threshold value $\sigma_{1}$.

2) The ratio of the area of the object in a sub-square to the area of the sub-square should be more than the threshold value $\sigma_{2}$ by formula (1).

$$
\sigma_{2}=\left(\frac{m}{\sigma_{1} M}\right)^{\rho}
$$

We can adjust the parameters $\sigma_{1}$ and $\rho$ to control the quadtree division. Let 
$G_{A}=\left\{\left(g_{1}^{A}, \operatorname{Area}_{1}^{A}\right),\left(g_{2}^{A}, \operatorname{Area}{ }_{2}^{A}\right), \ldots,\left(g_{N}^{A}, \operatorname{Arra}_{N}^{A}\right)\right\}$ and $G_{B}=\left\{\left(g_{1}^{B}, \operatorname{Area}_{1}^{B}\right),\left(g_{2}^{B}, \operatorname{Arra}_{2}^{B}\right), \ldots,\left(g_{M}^{B}, \operatorname{Area}{ }_{M}^{B}\right)\right\}$ be the sets of centroids of sub-squares covering the object $A$ and the object $B$ and their respective area of the object in the sub-square by formula (2) and formula (3). Let $\mathrm{Area}_{A}$ and $\mathrm{Area}_{B}$ be the areas of object the $A$ and the $B, N$ and $M$ be the numbers of $\mathrm{M} \mathrm{в} \mathrm{S}_{i}^{A}$ and $\mathrm{M} \mathrm{в} \mathrm{S}_{j}^{B}, \alpha_{i j}$ be the pairwise angle between $g_{i}{ }_{i}$ and $g_{j}^{B}$.

$$
\begin{aligned}
& \operatorname{Area}_{i}^{A}=\operatorname{Area}_{A} \cap M B S_{i}^{A} \\
& \operatorname{Area}_{j}^{B}=\operatorname{Area}_{B} \cap M B S_{j}^{B}
\end{aligned}
$$

Once the sets of sub-squares and their parameters are defined, we build the quadtree histogram by formula (4) and formula (5). Let $\tau$ be the step of quantization of the interval $[-\pi$, $\pi]$. We can adjust $\tau$ to handle the accuracy of the histogram. In this paper, let $\tau$ be 1 .

$$
\begin{gathered}
Q H_{\theta}(A, B)=\left\{\begin{array}{c}
+\operatorname{Area}_{i}^{A} * \operatorname{Area}_{j}^{B} \text { if } \alpha_{i j} \in\left[\theta-\frac{\tau}{2}, \theta+\frac{\tau}{2}\right] \\
0 \quad \text { else }
\end{array}\right. \\
Q H_{\theta}(A, B)=\frac{Q H_{\theta}(A, B)}{\sum_{\theta} Q H_{\theta}(A, B)}
\end{gathered}
$$

We construct a graphics database of quadtree histogram (GDQH) for our experiments, and it consists of 200 synthetic images of size $400 \times 400$ containing two objects with complicated or simple shape. We respectively generate angle-histogram and quadtree histogram as shown in Figure 1 for one image of GDQH in Figure 1a in $\mathrm{C}++$ programming language on a Pentium $3.19 \mathrm{GHz}$ PC with 2GB RAM. Figure 1b, Figure $1 \mathrm{c}$ and Figure $1 \mathrm{~d}$ respectively are the results of quadtree division with different values of $\sigma_{1}$ and $\rho$. Figure $1 \mathrm{f}$, Figure $1 \mathrm{~g}$ and Figure $1 \mathrm{~h}$ respectively are quadtree histograms with different values of $\sigma_{1}$ and $\rho$. These figures show that the smaller the values of $\sigma_{1}$ and $\rho$ are, the higher the accuracy of division is, and the more similar the shapes of angle-histogram and quadtree histogram are. As $\sigma_{1}=1 / M$, quadtree histogram is equal to angle-histogram. But Table 1 shows that the smaller the values of $\sigma_{1}$ and $\rho$ are, the higher the computational complexity of quadtree histogram is. In this paper, we define $\sigma_{1}=1 / 3$ and $\rho=1 / 3$ in order to get the high accuracy and the low computational complexity together.
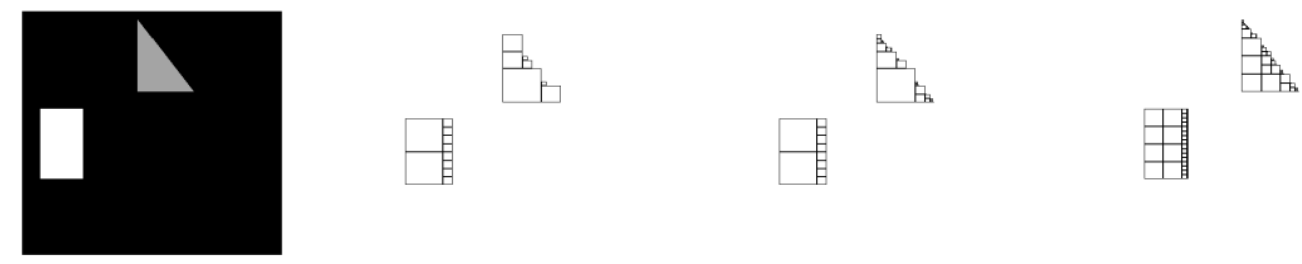

a) Original image

b) $\sigma_{1}=1 / 3$ and $\rho=1$

c) $\sigma_{1}=1 / 3$ and $\rho=1 / 3$

d) $\sigma_{1}=1 / 6$ and $\rho=1 / 3$ 


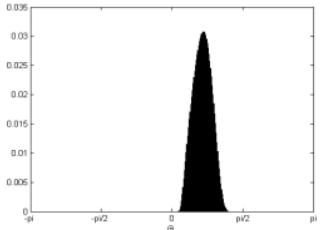

e) Angle-histogram

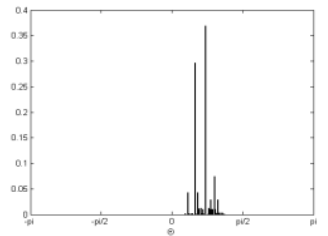

f) $\sigma_{1}=1 / 3$ and $\rho=1$

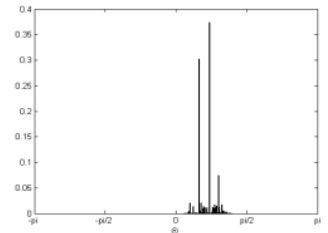

g) $\sigma_{1}=1 / 3$ and $\rho=1 / 3$

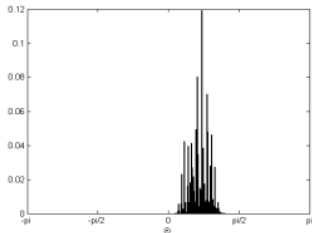

h) $\sigma_{1}=1 / 6$ and $\rho=1 / 3$

Figure 1. Quadtree Histogram

Table 1. CPU Time

\begin{tabular}{l|cccc}
\hline (s) & Angle-histogram & $\sigma_{1}=1 / 3$ and $\rho=1$ & $\sigma_{1}=1 / 3$ and $\rho=1 / 3$ & $\sigma_{1}=1 / 6$ and $\rho=1 / 3$ \\
\hline me of CPU & 3.258 & 0.008 & 0.011 & 0.023 \\
\hline
\end{tabular}

\subsection{The Judgment Approach of the Basic Directional Relation}

Directional relation is a qualitative concept $[7,8]$, so fuzzy set theory often is used for the judgment of directional relation. The directional relation between $p$ and $q$ is determined by the angle $\theta$ made by the line passing through the two points and the $\mathrm{x}$-axis. Directional relation is a fuzzy set whose membership functions are given by Gauss functions of $\theta$ in Figure 2, standard deviation is $\pi / 8$, mean values respectively are $-\pi,-\pi / 2,0, \pi / 2, \pi$.

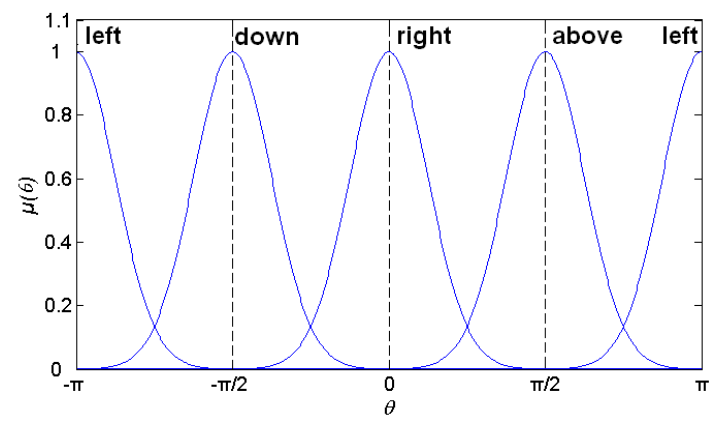

Figure 2. Gauss Membership Functions

An object generally is not a point, and we can consider the object as the set of points. Now we discuss this case. 1) Opposite directions weaken each other. Take Figure 3a for example, the gray object is somewhat above, below, to the right and to the left of the white object as well, but the judgment often is "the gray object is to the left of the white object". The reason is that above and below weaken each other, so the judgment do not include above or below. 2) Directional relation has the character of shift. In Figure $3 \mathrm{~b}$, the gray object although is to the right of the white object, but shifts downward. Considering these two characters, the availability degree and the certainty degree of directional relation are used for judging the basic directional relation. 


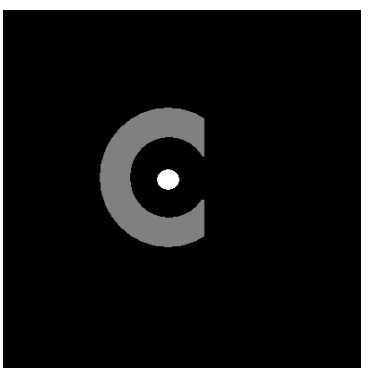

a)

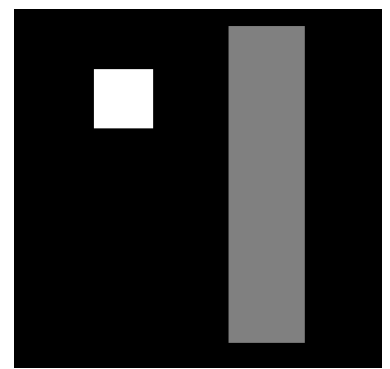

b)

Figure 3. Synthetic Images

Take direction right for example. Firstly, Quadtree histogram is divided into four quadrants: $\theta \in[-\pi,-\pi / 2], \theta \in[-\pi / 2,0], \theta \in[0, \pi / 2]$ and $\theta \in[\pi / 2, \pi]$, as shown in Figure 4. Secondly, we define the effective area and the weaken area. The effective area is the inner quadrants $(\theta \in[-\pi / 2,0]$ or $\theta \in[0, \pi / 2])$, and the weaken area is the outer quadrants $(\theta \in[-\pi,-$ $\pi / 2]$ or $\theta \in[\pi / 2, \pi])$. The effective area supports the proposition of direction right, but the weaken area weakens this proposition. Thirdly, we calculate the availability degree $b$ of directional relation by formula (6), and calculate the certainty degree $a$ of directional relation based on the angle $\alpha$ of shift by formula (7) and formula (8). Finally, the confidence degree $C$ is calculated by formula (9). By comparing $C$ of the four directions, we can determine the primary direction and the second direction of the basic directional relation. In these formulas, $\mu$ is the membership function of direction, and $f(\theta)$ is the histogram function.

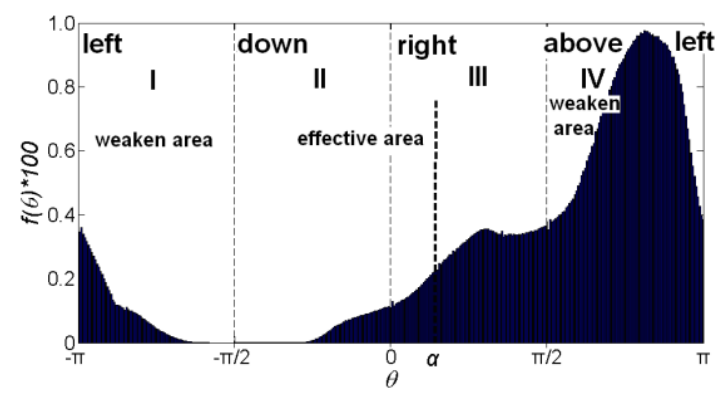

Figure 4. Effective Area and Weaken Area

$$
\begin{aligned}
& b_{r i g h t}=\frac{\left(\sum_{\theta} \mu_{r i g h t}(\theta) f(\theta)-\sum_{\theta} \mu_{\text {left }}(\theta) f(\theta)\right)}{\sum_{\theta} \mu_{r i g h t}(\theta) f(\theta)} \\
& \alpha_{r i g h t}=\frac{\sum_{\theta} \mu_{r i g h t}(\theta) f(\theta) \theta}{\sum_{\theta} \mu_{r i g h t}(\theta) f(\theta)} \\
& a_{r i g h t}=\mu\left(\alpha_{r i g h t}\right) \\
& C_{r i g h t}=a_{r i g h t} \times b_{r i g h t}
\end{aligned}
$$




\subsection{The Judgment Approach of the Special Directional Relation}

Firstly, we calculate the quad-tree histograms $Q H_{\theta}\left(M B S_{i}^{A}, B\right)$ of every sub-square $\mathrm{M} \mathrm{BS}_{i}^{A}$ to the reference object $B$. Secondly, we find the largest open interval $z_{1}$ and the second largest open interval $z_{2}$ of which value is zero. $\theta_{1}$ and $\theta_{2}$ respectively are the right borders of $z_{1}$ and $z_{2}$, as shown in Figure 5.

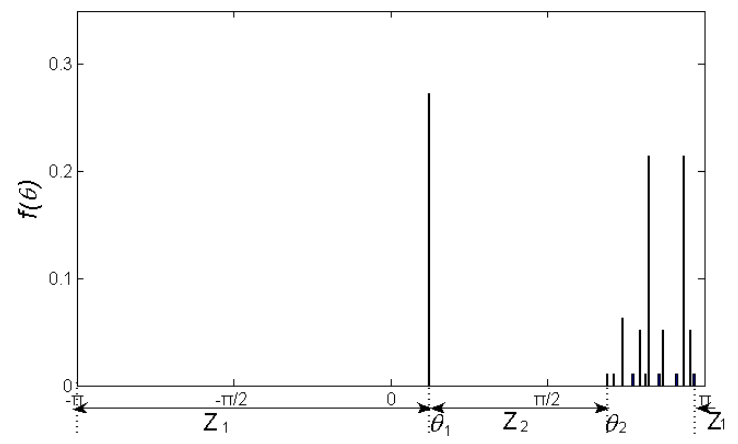

Figure 5. $z_{1}, z_{2}, \theta_{1}$ and $\theta_{2}$

Surround is one of the important special directional relation. In this paper we calculate the membership values $\mu_{\text {surround }}^{M B S_{i}^{A}}$ of surround between every sub-squares $M B S_{i}^{A}$ of the object $A$ and the object $B$ by formula (10) based on visual surroundedness theory, and then calculate the weighted average $\mu_{\text {surround }}$ of $\mu_{\text {surround }}^{M B i_{1}^{A}}$ as the membership value of the judgment "object $\mathrm{B}$ surround object A" by formula (11).

$$
\begin{aligned}
& \mu_{\text {surround }}^{M B S_{i}^{A}}\left(z_{i 1}\right)=\left\{\begin{array}{cc}
\cos ^{2} \frac{z_{i 1}}{2} & \text { if } 0 \leq z_{i 1} \leq \pi \\
0 & \text { ortherwise }
\end{array}\right. \\
& \mu_{\text {surround }}=\sum_{j=1}^{N} \mu_{\text {surround }}^{M B S S_{i 1}^{A}}\left(z_{i 1}\right) \times\left(\text { Area }_{i}^{A} / \text { Area }^{A}\right)
\end{aligned}
$$

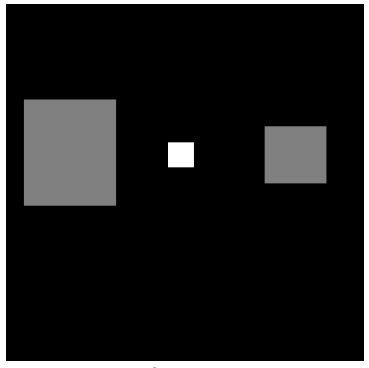

a)

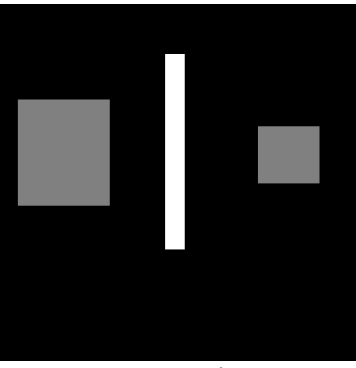

b)

Figure 6. Synthetic Images

Between is a ternary relation of the special directional relation. For example, $A$ is between $B_{1}$ and $B_{2}$. A simple approach firstly calculate the membership value $\mu_{\text {between }}^{M B S_{i}^{A}}$ of the judgment " $M B S_{i}{ }_{i}^{A}$ between $B_{1}$ and $B_{2}$ " by formula (12), and then calculate the weighted average $\mu_{\text {between }}$ of $\mu_{\text {between }}^{M B S_{i}^{A}}$ as the membership value of between by formula (13). But in many cases, this 
approach is not correct. For example, the white object is between the two gray objects in Figure 6b, but the judgment by above approach is not correct. But in Figure 6a the result is correct. As shown in Figure 6b, the parts of the white object are somewhat above or below the gray object, not between, so we should consider the weakening effect of symmetrical parts. According to this idea, we use formula (14) and formula (15) instead of formula (12) and formula (13).

$$
\begin{aligned}
& \mu_{\text {between }}^{M B S_{i}^{A}}\left(z_{i 1}, z_{i 2}\right)=\frac{z_{i 2}}{z_{i 1}} \\
& \mu_{\text {between }}=\sum_{i=1}^{N} \mu_{\text {between }}^{M B S_{i}^{A}}\left(z_{i 1}, z_{i 2}\right) \times\left(\text { Area }_{i}^{A} / \text { Area }^{A}\right) \\
& \mu_{\text {between }}^{M B S_{i}^{A}}\left(z_{i 1}, z_{i 2}\right)=\left\{\begin{array}{cc}
\frac{z_{i 2}}{z_{i 1}} & \text { if } \theta_{i 1}<\theta_{i 2} \\
2-\frac{z_{i 2}}{z_{i 1}} & \text { ortherwise }
\end{array}\right. \\
& \mu_{\text {between }}=\left\{\begin{array}{cc}
\mu_{\text {between }} & \text { if } \mu_{\text {between }}<1 \\
2-\mu_{\text {between }} & \text { ortherwise }
\end{array}\right.
\end{aligned}
$$

\section{Experimental Results}

In this section, we show partial experimental results. We respectively generate anglehistogram, F-histogram [21] and quadtree histogram for six images of GDQH in figure 7 in $\mathrm{C}++$ programming language on a Pentium $3.19 \mathrm{GHz}$ PC with 2GB RAM. Because the number of sub-squares is much smaller than the number of pixels, the computational complexity of quadtree histogram is much lower than angle-histogram, and generally lower than F-histogram, as shown in Table 2. In addition, we can get the following conclusions by experimental results. Firstly, the larger the area of the object is, the longer the CPU time is, whereas the shorter. For example, the CPU time of Figure $7 \mathrm{~b}$ is longer than Figure $7 \mathrm{a}$. Secondly, the longer the distance between objects is, the shorter the CPU time is, whereas the longer. For example, the CPU time of Figure 7c is shorter than Figure 7d. Thirdly, the more regular the object is, the shorter the CPU time is, whereas the longer. For example, the CPU time of Figure $7 \mathrm{f}$ is shorter than Figure $7 \mathrm{e}$.

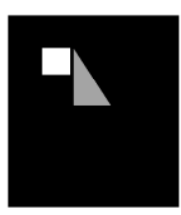

a)

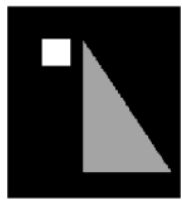

b)

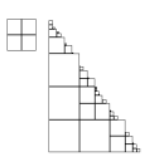

a')

b')
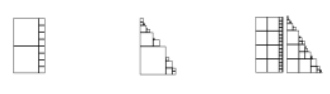

c')

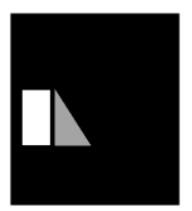

d)

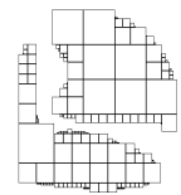

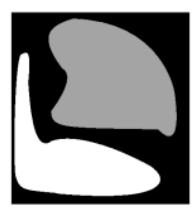

e)

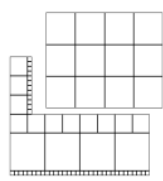

$\left.e^{\prime}\right)$

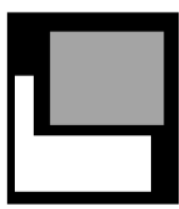

f) d') f') 


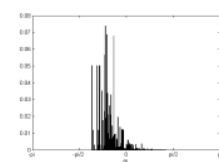

a")

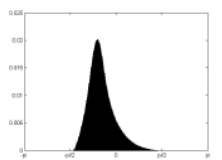

a"')

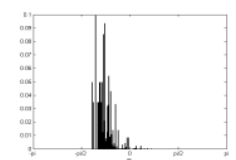

b")

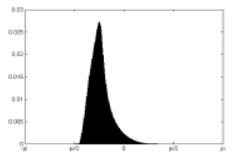

b"')

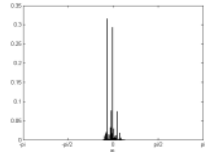

c")

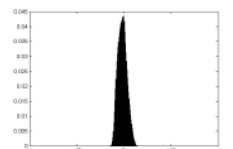

c"')

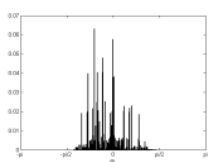

d")

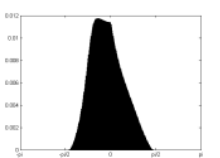

d"')

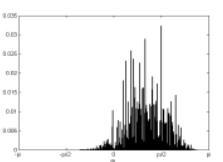

e")

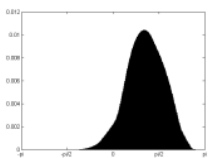

e"')

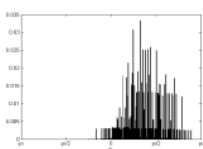

f')

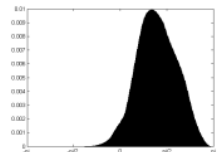

f"')

Figure 7. Examples

Table 2. CPU Time of Computation

\begin{tabular}{c|cccccc}
\hline$(\mathrm{s})$ & $\mathrm{a}$ & $\mathrm{b}$ & $\mathrm{c}$ & $\mathrm{d}$ & $\mathrm{e}$ & $\mathrm{f}$ \\
\hline Angle-histogram & 1.610 & 8.657 & 3.266 & 3.282 & 163.454 & 184.766 \\
F-histogram & 0.094 & 1.250 & 0.094 & 0.109 & 3.657 & 1.079 \\
Quad-tree histogram & 0.013 & 0.045 & 0.011 & 0.023 & 0.673 & 0.158 \\
\hline
\end{tabular}

We respectively use five methods ( $\mathrm{K}$ [9], $\mathrm{M}$ [10], $\mathrm{F}_{0}$ [22], $\mathrm{F}_{2}$ [22], Q) to judge the basic directional relation of eight images of GDQH in figure 8. The results are shown in table $3 . \mathrm{K}$ and $\mathrm{M}$ methods base on angle-histogram, and $\mathrm{F}_{0}$ and $\mathrm{F}_{2}$ methods base on F-histogram. $\mathrm{Q}$ method bases on quadtree histogram, and uses the new judgment approach in this paper. To determine a method whether good, we can use the notion of directional relation in article [23] to analyze the characteristics of judging directional relation with every method in the nonspecific application.

The judgment results of $\mathrm{K}$ and $\mathrm{M}$ often assign degree values to the four directions. It doesn't meet human's habits of describing directional relation with one or two directional terms as in Figure $8 \mathrm{f}$ and Figure $8 \mathrm{~g}$. The judgments of $\mathrm{F}_{0}, \mathrm{~F}_{2}$ and $\mathrm{Q}$ have only one or two degree values not 0 , so these methods are consistent with human cognition. When the object is narrow, the judgments of $\mathrm{K}, \mathrm{M}$ and $\mathrm{F}_{0}$ are possibly different from human cognition. Take Figure $8 \mathrm{~b}$ for example, the judgment of $\mathrm{K}, \mathrm{M}$ and $\mathrm{F}_{0}$ is "primary direction is above", but the judgment of human often is "primary direction is right". $F_{2}$ method considers the reason is that the other methods can't consider the effect of distance information to directional relation. $\mathrm{F}_{2}$ method considers that the close point pair makes more contribution to directional relation. So $\mathrm{F}_{2}$ method adds distance information to resolve this problem. In this paper, we consider that distance indirectly affect directional relation by changing the shape of histograms, and the reason of incorrect judgment of figure $8 \mathrm{~b}$ is that membership function of directional relation is not fit. We use Guass function instead of trigonal function or trigonometric function, because membership values about above as $\theta=\pi / 2$ should be much larger than it as $\theta=\pi / 4$. By Q method, we can get consistent judgment with human cognition about Figure $8 \mathrm{~b}$ and Figure 8d.

In summary, Q method can get no more than two directional terms and resolve the problem of the narrow object.

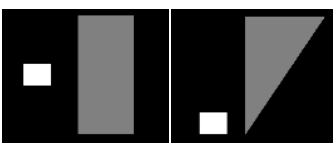

a)

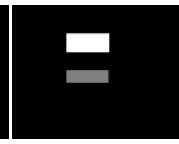

c)

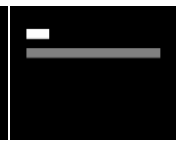

d)

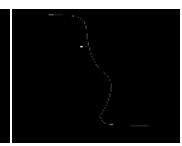

e)

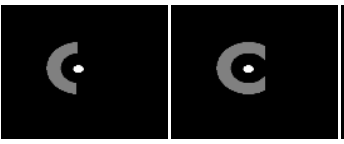

g)

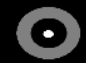

h)

Figure 8. Examples of the Basic Directional Relation 
Table 3. Results of the Basic Directional Relation

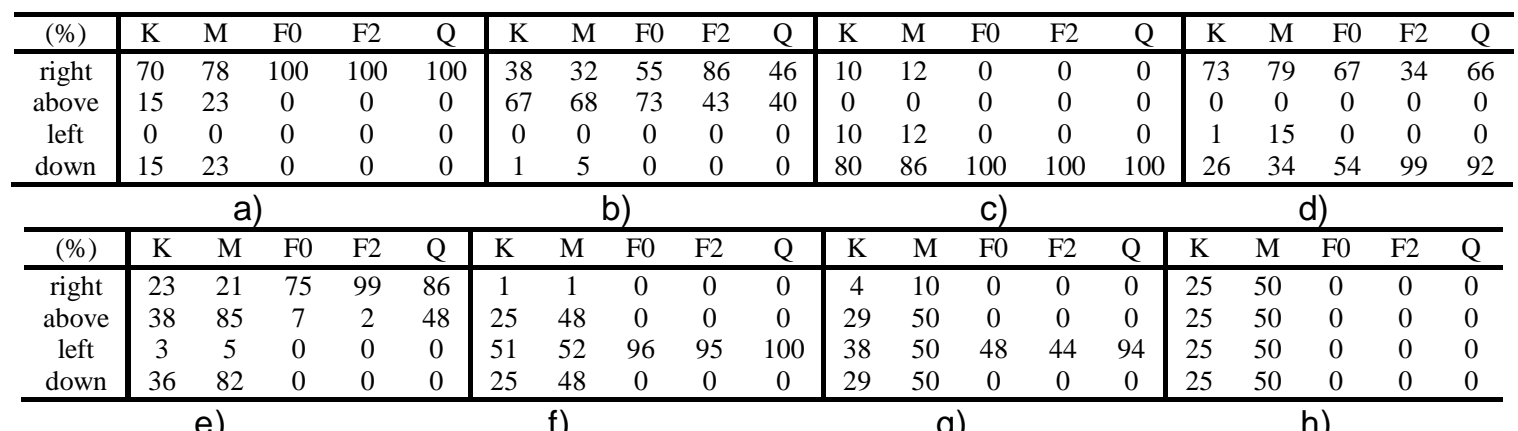

We respectively use three methods (K [9], FH [20], Q) to judge the special directional relation of twelve images of GDQH in Figure 9. The results are shown in Table 4.

Firstly, we analyze the judgment results of surround. The judgments of K, FH and Q to Figure 9a and Figure 9b are basically the same, but the judgments of FH to Figure 9c, figure 9d, Figure 9e and Figure 9f are not consistent with human cognition. That is because Fhistogram is difficult to reflect the direction surround of every part of the target object. The judgments of $\mathrm{K}$ and $\mathrm{Q}$ are consistent with human cognition, but the computational complexity of $\mathrm{Q}$ is much lower than $\mathrm{K}$.

Secondly, we analyze the judgment results of between. The judgments of K, FH and Q to Figure $9 \mathrm{~g}$, Figure $9 \mathrm{~h}$ and Figure $9 \mathrm{i}$ are basically the same, but the judgment of $\mathrm{K}$ to figure $9 \mathrm{i}$ is not consistent with human cognition obviously. That is because $\mathrm{K}$ method can't consider the weakening effect of symmetrical parts. The judgment values of Q to Figure 9j, Figure 9k and Figure 91 are greater than $\mathrm{FH}$, because Q method consider weakening effect of symmetrical parts.

In summary, Q method is more suitable to judging the special directional relation, and it is not restricted by the disconnected object.

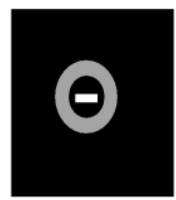

a)

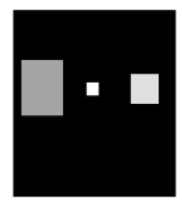

g)

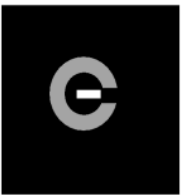

b)

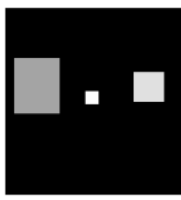

h)

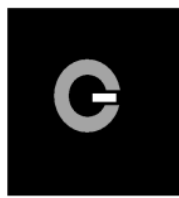

c)

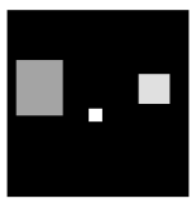

i)

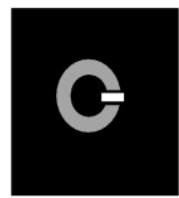

d)

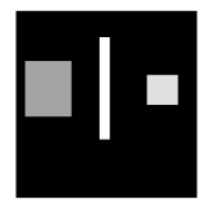

j)

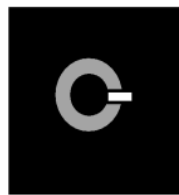

e)

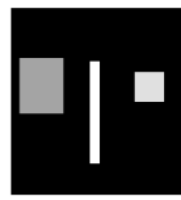

k)

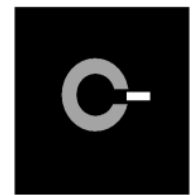

f)

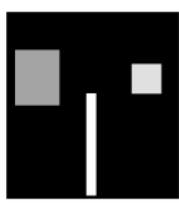

l)

Figure 9. Examples of the Special Directional Relation 
Table 4. Results of the Special Directional Relation

\begin{tabular}{c|cccccc|c|cccccc}
\hline$\mu_{\text {surround }}(\%)$ & $\mathrm{a}$ & $\mathrm{b}$ & $\mathrm{c}$ & $\mathrm{d}$ & $\mathrm{e}$ & $\mathrm{f}$ & $\mu_{\text {between }}(\%)$ & $\mathrm{g}$ & $\mathrm{h}$ & $\mathrm{i}$ & $\mathrm{j}$ & $\mathrm{k}$ & $\mathrm{L}$ \\
\hline $\mathrm{K}$ & 100 & 93 & 70 & 41 & 19 & 0 & $\mathrm{~K}$ & 99 & 76 & 51 & 54 & 51 & 29 \\
$\mathrm{FH}$ & 100 & 98 & 94 & 88 & 79 & 0 & $\mathrm{FH}$ & 98 & 71 & 45 & 95 & 55 & 22 \\
$\mathrm{Q}$ & 97 & 92 & 72 & 43 & 21 & 0 & $\mathrm{Q}$ & 99 & 74 & 49 & 99 & 66 & 39 \\
\hline
\end{tabular}

\section{Conclusion}

In this paper, a new formal model of directional relation based on quadtree histogram is firstly introduced. Firstly, we propose the quadtree histogram which inherits the characteristics of angle-histogram. The quadtree histogram can fully take into account the effects of shape, size and distance information to judging directional relation, and it also can ensure the high accuracy and the low complexity. Secondly, we propose a new judgment approach for the basic and special directional relation. The judgment results can be basically consistent with human cognition. The experimental results show that this model is in better harmony with human perception.

In future, we can apply this model in various domains, such as scene description, human-robot communication and retrieval.

\section{Acknowledgements}

This work is supported by "National Natural Science Foundation of China" (Grants No. 61302163 and No.61302105) "the Fundamental Research Funds for the Central Universities" and "Hebei Province Natural Science Foundation" (Grants No. F2012502015).

\section{References}

[1] A. U. Frank, "Qualitative spatial reasoning about cardinal directions", Austrian Conference on Artificial Intelligence, (1991), pp. 157-167.

[2] A. U. Frank, "Qualitative spatial reasoning: cardinal directions as an example", International Journal of Geographical Information Systems, vol. 10, no. 3, (1996), pp. 269-290.

[3] S. K. Chang, Q. S. Shi and C. W. Yan, "Iconic indexing by 2D strings", IEEE Transactions on Pattern Analysis and Machine Intelligence, vol. 9, no. 6, (1987), pp. 413-428.

[4] D. Papadias and T. Sellis, "Qualitative representation of spatial knowledge in two dimensional space", VLDB Journal, vol. 3, no. 4, (1994), pp. 479-516.

[5] R. Goyal and M. J. Egenhofer, "The direction-relation matrix: a representation for directions relations between extended spatial objects", The Annual Assembly and the Summer Retreat of University Consortium for Geographic Information Systems Science, (1997).

[6] H. W. Yan and R. Z. Guo, "Research on formal description model of directional relationships", Acta Geodaetica et Cartographica Sinica, vol. 32, no. 1, (2003), pp. 42-46.

[7] S. H. Du and Q. Wang, "Uncertain spatial relations", Journal of Image and Graphics, vol. 9, no. 5, (2004), pp. 539-546.

[8] S. H. Du, Q. Wang, Y. P. Yang and Z. J. Li, "Fuzzy description of spatial direction relations", Journal of Computer-aided Design \& Computer Graphics, vol. 17, no. 8, (2005), pp. 1744-1751.

[9] R. Krishnapuram, J. Keller and Y. Ma, "Quantitative analysis of properties and spatial relations of fuzzy image regions", IEEE Transactions on Fuzzy Systems, vol. 1, no. 3, (1993), pp. 222-233.

[10] K. Miyajima and A. Ralescu, "Spatial-organization in 2d segmented images: representation and recognition of primitive spatial relations", Fuzzy Sets and Systems, vol. 65, no. 2, (1994), pp. 100-105.

[11] Y. H. Wang and F. Makedon, "R-histogram: quantitative representation of spatial relations for similaritybased image retrieval", The Eleventh ACM International Conference on Multimedia, (2003), pp. 323-326. 
[12] Y. H. Wang, F. Makedon and A. Chakrabarti, "R*-histograms: efficient representation of spatial relations between objects of arbitrary topology", The Twelve ACM International Conference on Multimedia, (2004), pp. 356-359.

[13] P. Matsakis and L. Wendlling, "A new way to represent the relative position between areal objects", IEEE Transaction on Pattern Analysis and Machine Intelligence, vol. 21, no. 7, (1999), pp. 634-644.

[14] K. Zhang, K. P. Wang, X. J. Wang and Y. X. Zhong, "Spatial relations modeling based on visual area histogram", The 11th ACIS International Conference on Software Engineering, Artificial Intelligence, Networking and Parallel/Distributed Computing, (2010), pp. 323-326.

[15] P. Matsakis, "Combined extraction of directional and topological relationship information from 2D concave objects", Fuzzy Modeling with Spatial Information for Geographic Problems, (2005), pp. 15-40.

[16] K. C. Santosh, L. Wendling and B. Lamiroy "Using spatial relations for graphical symbol description", International Conference on Pattern Recognition, (2010), pp. 2041-2044.

[17] I. Bloch, "Fuzzy relative position between objects in image proceedings: new definition and properties based on a morphological approach", Int. J. of Uncertainty Fuzziness and Knowledge-Based Systems, vol. 7, no. 2, (1999), pp. 99-133.

[18] A. Rosenfeld and R. Klette, "Degree of adjacency or surroundedness", Pattern Recognition, vol. 18, no. 2, (1985), pp. 169-177.

[19] P. Matsakis and S. Andréfouět, "The fuzzy line between and surround", The 2002 IEEE International Conference on Fuzzy Systems, (2002), 1596-1601.

[20] M. Skubic, P. Matsakis, G. Chronis and J. Keller, "Generating multi-level linguistic spatial descriptions from range sensor readings using the histogram of forces", Autonomous Robots, vol. 14, no. 1, (2003), pp. 51-69.

[21] J. Ni and P. Matsakis, "Force histograms computed in O(NlongN)", International Conference on Pattern Recognition, pp. 1-4, (2008).

[22] P. Matsakis, J. M. Keller and L. Wending, "Linguistic descriptions of relative positions in images", IEEE Transactions on Systems, Man, and Cybernetics, vol. 31, no. 4, (2001), pp. 573-588.

[23] K. P. Gapp, "Basic meanings of spatial relations: Computation and evaluation in 3D space", American Association for AI National Conference, (1994), pp. 1393-1398.

\section{Author}

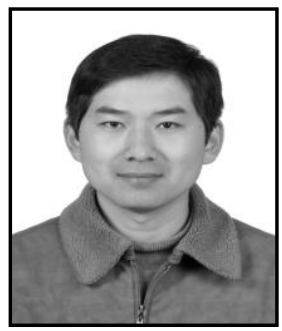

Ke Zhang, has been a teacher of North China Electric Power University. He received the PhD degree in signal and information processing in the Department of Computer Science at Beijing University of Posts and Telecommunications. He received the BSc degree (2003) and MSc degree (2006) in Department of Electronic and Communication Engineering at North China Electric Power University. His research interests include Computer Vision, Image Understanding, Natural Language Processing and Spatial Relation Description. 